\title{
Commentary Restoring normoglycaemia: not so harmless
} Jean-Charles Preiser

Department of General Intensive Care, University Hospital Centre, University of Liege, Domaine Universitaire du Sart Tilman - B 35, 4000 Liege, Belgium

Corresponding author: Jean-Charles Preiser, Jean-Charles.Preiser@chu.ulg.ac.be

Published: 28 February 2008

This article is online at http://ccforum.com/content/12/1/116

(c) 2008 BioMed Central Ltd

Critical Care 2008, 12:116 (doi:10.1186/cc6787)

\begin{abstract}
Three independent studies of tight glucose control were recently stopped prematurely due to an excess mortality in the intensive treatment arm. This commentary briefly discusses the potential mechanisms and reminds the potential benefits of physiological stress hyperglycemia.
\end{abstract}

In the closed world of intensive care medicine, the wave of enthusiasm triggered by the Leuven study [1] - which reported a dramatic improvement in the vital outcome of surgical intensive care unit patients after restoration of normoglycaemia by intensive insulin therapy - progressively vanished after the failure of several trials conducted to confirm these results in other institutions or settings.

Three different and independent multicentre studies designed to compare the outcome of patients randomised to a therapy designed to lower blood glucose versus conventional treatment were halted very recently, because of an increase in the rate of hypoglycaemia without a benefit in terms of mortality. More worrisome, a nonsignificant but consistent increase of the 28-day mortality was found in the first two trials conducted in intensive care - the Efficacy of Volume Substitution and Insulin Therapy in Severe Sepsis (VISEP) study [2] and the GluControl study [3]. In both trials, in which 537 septic patients and 1,101 critically ill patients were enrolled, intensive insulin therapy was titrated to reach blood glucose of $80-110 \mathrm{mg} / \mathrm{dl}$ (the normoglycaemic range). The results of the even larger Normoglycaemia in Intensive Care Evaluation and Survival Using Glucose Algorithm Regulation study [4] are awaited for the end of 2008, and will probably provide important additional information on the effects of intensive insulin therapy titrated to restore and maintain normoglycaemia in critically ill patients.

An announcement was recently made regarding a large National Institutes of Health-sponsored trial conducted in more than 10,000 outpatients with type II diabetes and at risk for heart attack and stroke followed in 77 North American centres [5]. The Action to Control Cardiovascular Risk in Diabetes (ACCORD) study was halted prematurely, however, because of an excess mortality in the intensive treatment arm. The sustained lowering of blood glucose was reflected by a fall below $6 \%$ of the percentage of glycosylated haemoglobin in the intensive treatment group, as planned per protocol.

Even though short-term and long-term mortality can hardly be integrated, these three major studies provide a consistent sound alarm signal against the concept of lowering blood glucose as a simple risk-free, harmless and potentially lifesaving therapeutic modality. Interestingly enough, the consistency of these findings is supported by the use of different therapeutic strategies - intravenous insulin in the VISEP and GluControl trials, and oral medications or subcutaneous insulin in the ACCORD study.

In fact, the use of the term normoglycaemia itself might be misleading when the range of normal blood glucose was defined in fasting healthy individuals and is mainly used to exclude the diagnosis of diabetes. In critically ill patients or in diabetic patients, such a blood glucose range $(80-110 \mathrm{mg} / \mathrm{dl})$ may not be normal, or desirable. The possible reasons for the increased mortality after lowering of blood glucose by intensive treatment can only be speculated, prior to a more detailed analysis of the causes of deaths. Nonetheless, inappropriately low glucose levels can induce a shortage of the provision of glucose, the predominant source of energy useable by the myocardium during ischaemia, and neuroglycopenia in cases of cerebral injury $[6,7]$. In both situations, the outcome of patients can be directly endangered.

The human body has actually developed physiological metabolic responses to survive stressful situations. Increased glucose turnover, and hence increased blood glucose concentrations, plays a major role in this response. We should 
probably bear these adaptive mechanisms in mind when developing new therapeutic strategies.

\section{Competing interests}

The author declares that they have no competing interests.

\section{References}

1. van den Berghe $G$, Wouters $P$, Weekers F, Verwaest $C$, Bruyninckx $F$, Schetz M, Vlasselaers D, Ferdinande P, Lauwers $P$, Bouillon R: Intensive insulin therapy in the critically ill patients. $N$ Engl J Med 2001, 345:1359-1367.

2. Brunkhorst FM, Engel C, Bloos F, Meier-Hellmann A, Ragaller M, Weiler N, Moerer O, Gruendling M, Oppert M, Grond S, Olthoff D, Jaschinski U, John S, Rossaint R, Welte T, Schaefer M, Kern P, Kuhnt E, Kiehntopf M, Hartog C, Natansori C, Loeffler M, Reinhart K, for the German Competence Network Sepsis (SepNet): Intensive insulin therapy and pentastarch resuscitation in severe sepsis. N Engl J Med 2008, 358:125-139.

3. Devos P, Preiser JC, Melot C, on behalf of the GluControl Steering Committee: Impact of tight glucose control by intensive insulin therapy on ICU mortality and the rate of hypoglycaemia: final results of the GluControl study. Intensive Care Med 2007, 33:S189.

4. NICE-SUGAR Study [http://clinicaltrials.gov/ct/show/ NCT00220987]

5. ACCORD Study Announcement [http://www.accordtrial.org]

6. Vespa P, Boonyaputthikul R, McArthur DL, Miller C, Etchepare M, Bergsneider M, Glenn T, Martin N, Hovda D: Intensive insulin therapy reduces microdialysis glucose values without altering glucose utilization or improving the lactate/pyruvate ratio after traumatic brain injury. Crit Care Med 2006, 34:850-856.

7. Schlenk F, Graetz D, Nagel A, Schmidt M, Sarrafzadeh AS: Insulin-related decreawse in cerebral glucose despite normoglycemia in aneurismal subarachnoid hemorrhage. Crit Care 2008, 12:R9. 